\title{
Strong differential subordinations and superordinations obtained with some new integral operators
}

Georgia Irina Oros

"Correspondence:
georgia_oros_ro@yahoo.co.uk
Department of Mathematics,
University of Oradea, Str.
Universităţii, No. 1, Oradea, 410087,
Romania

\begin{abstract}
In this paper we study certain strong differential subordinations and superordinations obtained by using some new integral operators introduced in (Oros and Oros in Differential subordinations obtained with some new integral operator (to appear)). MSC: $30 \mathrm{C} 80 ; 30 C 45 ; 30 C 20 ; 34 \mathrm{~A} 40$

Keywords: analytic function; univalent function; convex function; strong differential subordination and superordination; best dominant; best subordinant
\end{abstract}

\section{Introduction and preliminaries}

The concept of differential subordination was introduced in [1,2] and developed in [3] by Miller and Mocanu. The concept of differential superordination was introduced in [4] like a dual problem of the differential subordination by Miller and Mocanu and developed in [5]. The concept of strong differential subordination was introduced in [6] by Antonino and Romaguera and developed in [7-17]. The concept of strong differential superordination was introduced in [18] like a dual concept of the strong differential subordination and developed in [19-21].

In [8] the author defines the following classes:

Denote by $\mathcal{H}(U \times \bar{U})$ the class of analytic functions in $U \times \bar{U}$, where

$$
U=\{z \in \mathbb{C}:|z|<1\}, \quad \bar{U}=\{z \in \mathbb{C}:|z| \leq 1\}, \quad \partial U=\{z \in \mathbb{C}:|z|=1\} .
$$

For $a \in \mathbb{C}$ and $n \in \mathbb{N}^{*}$, we denote by

$$
\mathcal{H} \xi[a, n]=\left\{f(z, \xi) \in \mathcal{H}(U \times \bar{U}): f(z, \xi)=a+a_{n}(\xi) z^{n}+a_{n+1}(\xi) z^{n+1}+\cdots\right\}
$$

with $z \in U, \xi \in \bar{U}, a_{k}(\xi)$ holomorphic functions in $\bar{U}, k \geq n$. Let

$$
A \xi_{n}=\left\{f(z, \xi) \in \mathcal{H}(U \times \bar{U}): f(z, \xi)=z+a_{n+1}(\xi) z^{n+1}+\cdots\right\}
$$

with $z \in U, \xi \in \bar{U}, a_{k}(\xi)$ holomorphic functions in $\bar{U}, k \geq n+1$, and $A \xi_{1}=A \xi$,

$$
\begin{aligned}
& \mathcal{H} \xi_{u}(U)=\{f(z, \xi) \in \mathcal{H} \xi[a, n]: f(z, \xi) \text { is univalent in } U \text { for all } \xi \in \bar{U}\} \\
& S \xi=\left\{f(z, \xi) \in A \xi_{n}: f(z, \xi) \text { univalent in } U \text { for all } \xi \in \bar{U}\right\}
\end{aligned}
$$

( 2013 Oros; licensee Springer. This is an Open Access article distributed under the terms of the Creative Commons Attribution License (http://creativecommons.org/licenses/by/2.0), which permits unrestricted use, distribution, and reproduction in any medium, provided the original work is properly cited. 
denote the class of univalent functions in $\mathcal{H}(U \times \bar{U})$,

$$
S^{*} \xi=\left\{f(z, \xi) \in A \xi: \operatorname{Re} \frac{z f_{z}^{\prime}(z, \xi)}{f(z, \xi)}>0, z \in U, \text { for all } \xi \in \bar{U}\right\}
$$

denote the class of normalized starlike functions in $U \times \bar{U}$,

$$
K \xi=\left\{f(z, \xi) \in A \xi: \operatorname{Re}\left(\frac{z f_{z}^{\prime \prime}(z, \xi)}{f_{z}^{\prime}(z, \xi)}+1\right) \geq 0, z \in U, \text { for all } \xi \in \bar{U}\right\}
$$

denote the class of normalized convex functions in $U \times \bar{U}$.

Let $A(p) \xi$ denote the subclass of the functions $f(z, \xi) \in \mathcal{H}(U \times \bar{U})$ of the form

$$
f(z, \xi)=z^{p}+\sum_{k=p+1}^{\infty} a_{k}(\xi) z^{k}, \quad p \in N, z \in U, \text { for all } \xi \in \bar{U}, \text { and } \operatorname{set} A(1) \xi=A \xi .
$$

To prove our main results, we need the following definitions and lemmas.

Definition 1.1 $[7,18]$ Let $F(z, \xi)$ and $f(z, \xi)$ be members of $H(U \times \bar{U})$. The function $f(z, \xi)$ is said to be strongly subordinate to $F(z, \xi)$, or $F(z, \xi)$ is said to be strongly superordinate to $f(z, \xi)$ if there exists a function $w$ analytic in $U$, with $w(0)=0$ and $|w(z)|<1$ such that $f(z, \xi)=F(w(z), \xi)$ for all $\xi \in \bar{U}$. In such a case, we write $f(z, \xi) \prec \prec F(z, \xi), z \in U, \xi \in \bar{U}$. If $F(z, \xi)$ is univalent, then $f(z, \xi) \prec \prec F(z, \xi)$ if and only if $F(0, \xi)=f(0, \xi)$ and $f(U \times \bar{U}) \subset$ $F(U \times \bar{U})$.

Remark 1.1 If $F(z, \xi) \equiv F(z)$ and $f(z, \xi) \equiv f(z)$, then the strong differential subordination or superordination becomes the usual notions of differential subordination and superordination, respectively.

Definition 1.2 [7] We denote by $Q_{\xi}$ the set of functions $q(\cdot, \xi)$ that are analytic and injective, as functions of $z$ on $\bar{U} \backslash E(q(z, \xi))$, where $E(q(z, \xi))=\left\{\zeta \in \partial U: \lim _{z \rightarrow \zeta} q(z, \xi)=\infty\right\}$, and are such that $q^{\prime}(\zeta, \xi) \neq 0$ for $\zeta \in \partial U \backslash E(q(z, \xi)), \xi \in \bar{U}$. The subclass of $Q_{\xi}$ for which $q(0, \xi)=a$ is denoted by $Q_{\xi}(a)$.

Let $\Psi: \mathbb{C}^{3} \times U \times \bar{U} \rightarrow \mathbb{C}$, and let $h(z, \xi)$ be univalent in $U$ for all $\xi \in \bar{U}$. If $p(z, \xi)$ is analytic in $U \times \bar{U}$ and satisfies the (second-order) strong differential subordination

$$
\Psi\left(p(z, \xi), z p_{z}^{\prime}(z, \xi), z^{2} p_{z^{2}}^{\prime \prime}(z, \xi) ; z, \xi\right) \prec \prec h(z, \xi), \quad z \in U, \xi \in \bar{U}
$$

then $p(z, \xi)$ is called a solution of the strong differential subordination.

The univalent function $q(z, \xi)$ is called a dominant of the solutions of the strong differential subordination, or simply a dominant, if $p(z, \xi) \prec \prec q(z, \xi)$ for all $p(z, \xi)$ satisfying (1.1). A dominant $\widetilde{q}(z, \xi)$ that satisfies $\widetilde{q}(z, \xi) \prec \prec q(z, \xi)$ for all dominants $q(z, \xi)$ of (1.1) is said to be the best dominant of (1.1). Note that the best dominant is unique up to a rotation of $U \times \bar{U}$.

Let $\varphi: \mathbb{C}^{3} \times U \times \bar{U} \rightarrow \mathbb{C}$, and let $h(z, \xi)$ be analytic in $U \times \bar{U}$. If $p(z, \xi)$ and $\varphi(p(z, \xi)$, $\left.z p_{z}^{\prime}(z, \xi), z^{2} p_{z^{2}}^{\prime \prime}(z, \xi) ; z, \xi\right)$ are univalent in $U$ for all $\xi \in \bar{U}$ and satisfy the (second-order) strong differential superordination,

$$
h(z, \xi) \prec \prec \varphi\left(p(z, \xi), z p_{z}^{\prime}(z, \xi), z^{2} p_{z^{2}}^{\prime \prime}(z, \xi) ; z, \xi\right), \quad z \in U, \xi \in \bar{U},
$$


then $p(z, \xi)$ is called a solution of the strong differential superordination. An analytic function $q(z, \xi)$ is called a subordinant of the solutions of the strong differential superordination, or more simple a subordinant if $q(z, \xi) \prec \prec p(z, \xi)$ for all $p(z, \xi)$ satisfying $\left(1.1^{\prime}\right)$. A univalent subordinant $\widetilde{q}(z, \xi)$ that satisfies $q(z, \xi) \prec \prec \widetilde{q}(z, \xi)$ for all subordinants $q(z, \xi)$ of $\left(1.1^{\prime}\right)$ is said to be the best subordinant. Note that the best subordinant is unique up to a rotation of $U \times \bar{U}$.

We rewrite the operators defined in [22] for the classes presented earlier as follows.

Definition 1.3 [22] $\operatorname{For} f(z, \xi) \in A \xi_{n}, n \in N^{*}, m \in N, \gamma \in C$, let $L_{\gamma}$ be the integral operator given by $L_{\gamma}: A \xi_{n} \rightarrow A \xi_{n}$,

$$
\begin{aligned}
& L_{\gamma}^{0} f(z, \xi)=f(z, \xi) \\
& L_{\gamma}^{1} f(z, \xi)=\frac{\gamma+1}{z^{\gamma}} \int_{0}^{z} L_{\gamma}^{0} f(t, \xi) t^{\gamma-1} d t, \\
& L_{\gamma}^{2} f(z, \xi)=\frac{\gamma+1}{z^{\gamma}} \int_{0}^{z} L_{\gamma}^{1} f(t, \xi) t^{\gamma-1} d t, \quad \ldots, \\
& L_{\gamma}^{m} f(z, \xi)=\frac{\gamma+1}{z^{\gamma}} \int_{0}^{z} L_{\gamma}^{m-1} f(t, \xi) \cdot t^{\gamma-1} d t .
\end{aligned}
$$

By using Definition 1.3, we can prove the following properties for this integral operator: For $f(z, \xi) \in A \xi_{n}, n \in \mathbb{N}^{*}, m \in \mathbb{N}, \gamma \in \mathbb{C}$, we have

$$
L_{\gamma}^{m} f(z, \xi)=z+\sum_{k=n+1}^{\infty} \frac{(\gamma+1)^{m}}{(\gamma+k)^{m}} \cdot a_{k}(\xi) z^{k}, \quad z \in U, \xi \in \bar{U},
$$

and

$$
z \cdot\left[L_{\gamma}^{m} f(z, \xi)\right]_{z}^{\prime}=(\gamma+1) L_{\gamma}^{m-1} f(z, \xi)-\gamma L_{\gamma}^{m} f(z, \xi), \quad z \in U, \xi \in \bar{U} .
$$

Definition 1.4 [22] For $p \in N, m \in N, f(z, \xi) \in A(p) \xi$, let $H$ be the integral operator given by $H: A(p) \xi \rightarrow A(p) \xi$,

$$
\begin{aligned}
& H^{0} f(z, \xi)=f(z, \xi), \\
& H^{1} f(z, \xi)=\frac{p+1}{z} \int_{0}^{z} H^{0} f(t, \xi) d t, \\
& H^{2} f(z, \xi)=\frac{p+1}{z} \int_{0}^{z} H^{1} f(t, \xi) d t, \\
& H^{m} f(z, \xi)=\frac{p+1}{z} \int_{0}^{z} H^{m-1} f(t, \xi) d t .
\end{aligned}
$$

By using Definition 1.4, we can prove the following properties for this integral operator: For $f(z, \xi) \in A(p) \xi, m \in \mathbb{N}, p \in \mathbb{N}$, we have

$$
H^{m} f(z, \xi)=z^{p}+\sum_{k=p+1}^{\infty} \frac{(p+1)^{m}}{(p+k)^{m}} a_{k}(\xi) z^{k}, \quad z \in U, \xi \in \bar{U}
$$


and

$$
z\left[\cdot H^{m} f(z, \xi)\right]^{\prime}=(p+1) H^{m-1} f(z, \xi)-H^{m} f(z, \xi), \quad z \in U, \xi \in \bar{U}
$$

We rewrite the following lemmas for the classes presented earlier (the proofs are similar to those found in [6]).

Lemma 1.1 [3, Th. 3.4, p.132] Let the function $q(z, \xi)$ be univalent in $U$ for all $\zeta \in \bar{U}$, and let $\theta$ and $\varphi$ be analytic in a domain D containing $q(U \times \bar{U})$ with $q(\omega, \xi) \neq 0$ when $\omega \in q(U \times \bar{U})$.

Set $Q(z, \xi)=z q_{z}^{\prime}(z, \xi) \cdot \varphi(q(z, \xi))$ and $h(z, \xi)=\theta(q(z, \xi))+Q(z, \xi)$. Suppose that

(i) $Q(z, \xi)$ is starlike univalent in $U$ for all $\xi \in \bar{U}$,

(ii) $\operatorname{Re} \frac{z h_{z}^{\prime}(z, \xi)}{Q(z, \xi)}>0, z \in U$ for all $\xi \in \bar{U}$.

If $p(z, \xi)$ is analytic in $U \times \bar{U}$ with $p(0, \xi)=q(0, \xi), p(U \times \bar{U}) \subseteq D$ and

$$
\theta(p(z, \xi))+z p^{\prime}(z, \xi) \cdot \varphi(p(z, \xi)) \prec \prec \theta(q(z, \xi))+z q^{\prime}(z, \xi) \cdot \varphi(q(z, \xi))
$$

then $p(z, \xi) \prec \prec q(z, \xi), z \in U, \xi \in \bar{U}$ and $q(z, \xi)$ is the best strong dominant.

Lemma 1.2 [5, Corollary 1.1] Let $\alpha, \beta, \gamma \in \mathbb{C}$, and let $h(z, \xi)$ be convex in $U$ for all $\zeta \in \bar{U}$, with $h(0, \xi)=a$ and $q(z, \xi) \prec \prec h(z, \xi), z \in U, \xi \in \bar{U}$. Suppose that the differential equation $q(z, \xi)+\frac{z q_{z}^{\prime}(z, \xi)}{q(z, \xi)}=h(z, \xi)$ has a univalent solution $q(z, \xi)$ that satisfies $q(0, \xi)=a$.

If $p(z, \xi) \in[a, 1] \cap Q_{\xi}$ and $p(z, \xi)+\frac{z p_{z}^{\prime}(z, \xi)}{\beta p(z, \xi)+\gamma}$ is univalent in $U$ for all $\xi \in \bar{U}$, then $h(z, \xi) \prec \prec$ $p(z, \xi)+\frac{z p_{z}^{\prime}(z, \xi)}{\beta p(z, \xi)+\gamma}$ implies $q(z, \xi) \prec \prec p(z, \xi), z \in U, \xi \in \bar{U}$. The function $q(z, \xi)$ is the best subordinant.

\section{Main results}

We first give results related to strong differential subordinations.

Theorem 2.1 Let $q(z, \xi)$ be univalent in $U$ for all $\xi \in \bar{U}$, with $q(0, \xi)=1$ and $q(z, \xi) \neq 0$, and suppose that

(j) $\operatorname{Re} q(z, \xi)>0$,

(jj) $\operatorname{Re}\left(1+\frac{z q_{z^{2}}^{\prime \prime}(z, \xi)}{q_{z}^{\prime}(z, \xi)}-\frac{z q_{z}^{\prime}(z, \xi)}{q(z, \xi)}\right)>0$.

Let $n \in \mathbb{N}^{*}, \gamma \in \mathbb{C}, f(z, \xi) \in A \xi_{n}$ and

$$
\begin{aligned}
& \frac{L_{\gamma}^{m} f(z, \xi) \cdot\left[L_{\gamma}^{m} f(z, \xi)\right]_{z}^{\prime}}{z}+\frac{z\left[L_{\gamma}^{m} f(z, \xi)\right]_{z^{2}}^{\prime \prime}}{\left[L_{\gamma}^{m} f(z, \xi)\right]_{z}^{\prime}}+\frac{\left[L_{\gamma}^{m} f(z, \xi)\right]_{z}^{\prime}}{L_{\gamma}^{m} f(z, \xi)}-1 \\
& \prec \prec q(z, \xi)+\frac{z q_{z}^{\prime}(z, \xi)}{q(z, \xi)}, \quad z \in U, \xi \in \bar{U},
\end{aligned}
$$

then

$$
\frac{\left[L_{\gamma}^{m} f(z, \xi)\right]_{z}^{\prime} \cdot L_{\gamma}^{m} f(z, \xi)}{z} \prec \prec q(z, \xi), \quad z \in U, \xi \in \bar{U},
$$

and $q(z, \xi)$ is the best dominant. 
Proof We let

$$
p(z, \xi)=\frac{\left[L_{\gamma}^{m} f(z, \xi)\right]_{z}^{\prime} \cdot L_{\gamma}^{m} f(z, \xi)}{z}, \quad z \in U, \xi \in \bar{U}
$$

Using (1.2) in (2.2), we have

$$
\begin{aligned}
p(z, \xi) & =\frac{\left[z+\sum_{k=n+1}^{\infty} \frac{(\gamma+1)^{m}}{(\gamma+k)^{m}} a_{k}(\xi) z^{k}\right]^{\prime}\left[z+\sum_{k=n+1}^{\infty} \frac{(\gamma+1)^{m}}{(\gamma+k)^{m}} a_{k}(\xi) z^{k}\right]}{z} \\
& =1+A_{n}(\xi) z^{n}+\cdots,
\end{aligned}
$$

and since $p(0, \xi)=1$, we obtain that $p(z, \xi) \in \xi[1, n]$.

Differentiating (2.2), and after a short calculus, we obtain

$$
p(z, \xi)+\frac{z p_{z}^{\prime}(z, \xi)}{p(z, \xi)}=\frac{\left[L_{\gamma}^{m} f(z, \xi)\right]_{z}^{\prime} \cdot L_{\gamma}^{m} f(z, \xi)}{z}+\frac{z\left[L_{\gamma}^{m} f(z, \xi)\right]_{z^{2}}^{\prime \prime}}{\left[L_{\gamma}^{m} f(z, \xi)\right]_{z}^{\prime}}+\frac{z\left[L_{\gamma}^{m} f(z, \xi)\right]_{z}^{\prime}}{L_{\gamma}^{m} f(z, \xi)}-1
$$

Using (2.3) in (2.1), the strong differential subordination (2.1) becomes

$$
p(z, \xi)+\frac{z p_{z}^{\prime}(z, \xi)}{p(z, \xi)} \prec \prec q(z, \xi)+\frac{z q_{z}^{\prime}(z, \xi)}{q(z, \xi)}, \quad z \in U, \xi \in \bar{U}
$$

In order to prove the theorem, we shall use Lemma 1.1. For that, we show that the necessary conditions are satisfied. Let the functions $\Theta: \mathbb{C} \rightarrow \mathbb{C}$ and $\varphi: \mathbb{C} \rightarrow \mathbb{C}$, with

$$
\Theta(\omega)=\omega
$$

and

$$
\varphi(\omega)=\frac{1}{\omega}, \quad \varphi(w) \neq 0 .
$$

We check the conditions from the hypothesis of Lemma 1.1. Using (2.6), we have

$$
Q(z, \xi)=\frac{z q_{z}^{\prime}(z, \xi)}{q(z, \xi)}
$$

Differentiating (2.7), and after a short calculus, we obtain

$$
\frac{z Q_{z}^{\prime}(z, \xi)}{Q(z, \xi)}=1+\frac{z q_{z^{2}}^{\prime \prime}(z, \xi)}{q_{z}^{\prime}(z, \xi)}-\frac{z q_{z}^{\prime}(z, \xi)}{q_{z}(z, \xi)}
$$

Using (ji) in (2.8), we have

$$
\operatorname{Re} \frac{z Q_{z}^{\prime}(z, \xi)}{Q(z, \xi)}>0, \quad z \in U, \xi \in \bar{U}
$$

hence the function $Q(z, \xi)$ is starlike in $U$ for all $\xi \in \bar{U}$. Using (2.5) we have

$$
h(z, \xi)=\theta(q(z, \xi))+Q(z, \xi)=q(z, \xi)+Q(z, \xi) .
$$


Differentiating (2.10) and using (2.7), after a short calculus, we obtain

$$
\operatorname{Re} \frac{z h_{z}^{\prime}(z, \xi)}{Q(z, \xi)}=\operatorname{Re}\left[q(z, \xi)+\frac{z Q_{z}^{\prime}(z, \xi)}{Q(z, \xi)}\right]
$$

Using (j) and (2.9) in (2.11), we have $\operatorname{Re} \frac{z h_{z}^{\prime}(z, \xi)}{Q(z, \xi)}>0, z \in U, \xi \in \bar{U}$. Using (2.5) and (2.6), we get

$$
\begin{array}{ll}
\theta(p(z, \xi))=p(z, \xi), & \varphi(p(z, \xi))=\frac{1}{p(z, \xi)}, \\
Q(q(z, \xi))=q(z, \xi), \quad & \varphi(q(z, \xi))=\frac{1}{q(z, \xi)},
\end{array}
$$

and the strong differential subordination (2.1) becomes

$$
Q(p(z, \xi))+z p^{\prime}(z, \xi) \cdot \varphi(p(z, \xi)) \prec \prec \theta(q(z, \xi))+z q^{\prime}(z, \xi) \cdot \varphi(q(z, \xi))
$$

Using Lemma 1.1, we obtain

$$
p(z, \xi) \prec \prec q(z, \xi), \quad \text { i.e. } \quad \frac{\left[L_{\gamma}^{m} f(z, \xi)\right]_{z}^{\prime} \cdot L_{\gamma}^{m} f(z, \xi)}{z} \prec \prec q(z, \xi), \quad z \in U, \xi \in \bar{U},
$$

and $q(z, \xi)$ is the best dominant.

Theorem 2.2 Let $q(z, \xi)$ be univalent in $U$ for all $\xi \in \bar{U}$, with $q(0, \xi)=p-1$ and $q(z, \xi) \neq-1$, $z \in U$, for all $\xi \in \bar{U}$, and suppose that

(l) $\operatorname{Re} q(z, \xi)>-1$,

(ll) $\operatorname{Re}\left[1+\frac{z q_{2}^{\prime \prime}(z, \xi)}{q_{z}^{\prime}(z, \xi)}-\frac{z q_{z}^{\prime}(z, \xi)}{1+q(z, \xi)}\right]>0, z \in U, \xi \in \bar{U}$.

Let $p \in \mathbb{N}, f(z, \xi) \in A(p) \xi$ and

$$
\frac{z\left(H^{m} f(z, \xi)\right)_{z^{2}}^{\prime \prime}}{\left(H^{m} f(z, \xi)\right)_{z}^{\prime}}+\frac{\left(H^{m} f(z, \xi)\right]_{z}^{\prime}}{z^{p-1}}-p+1 \prec \prec q(z, \xi)+1+\frac{z q_{z}^{\prime}(z, \xi)}{q(z, \xi)+1}, \quad z \in U, \xi \in \bar{U}
$$

then $\frac{z\left(H^{m} f(z, \xi)\right)_{z}^{\prime}}{z^{p-1}}-1 \prec \prec q(z, \xi)$, and $q(z, \xi)$ is the best dominant.

Proof We let

$$
1+p(z, \xi)=\frac{\left(H^{m} f(z, \xi)\right)_{z}^{\prime}}{z^{p-1}}, \quad z \in U, \xi \in \bar{U}
$$

From (1.4), we have

$$
p(z, \xi)+1=\frac{\left(z^{p}+\sum_{k=p+1}^{\infty} \frac{(p+1)^{m}}{(p+k)^{m}} a_{k}(\xi) z^{k}\right)^{\prime}}{z^{p-1}}=p+\sum_{k=p+1}^{\infty} \frac{(p+1)^{m}}{(p+k)^{m}} a_{k}(\xi) k z .
$$

Since $p(0, \xi)=p-1$, we obtain that $p(z, \xi) \in \mathcal{H} \xi[p-1,1]$. Differentiating (2.13), and after a short calculus, we obtain

$$
\frac{z\left(H^{m} f(z, \xi)\right)_{z}^{\prime \prime}}{\left(H^{m} f(z, \xi)\right)_{z}^{\prime}}+\frac{\left(H^{m} f(z, \xi)\right]_{z}^{\prime}}{z^{p-1}}-p+1=p(z, \xi)+1+\frac{z p_{z}^{\prime}(z, \xi)}{p(z, \xi)+1}
$$


Using (2.14) in (2.12), the strong differential subordination becomes

$$
p(z, \xi)+1+\frac{z p_{z}^{\prime}(z, \xi)}{p(z, \xi)+1} \prec \prec q(z, \xi)+1+\frac{z q_{z}^{\prime}(z, \xi)}{q(z, \xi)+1}, \quad z \in U, \xi \in \bar{U} .
$$

In order to prove the theorem, we shall use Lemma 1.1. For that, we show that the necessary conditions are satisfied. Let the functions $\Theta: \mathbb{C} \rightarrow \mathbb{C}$ and $\varphi: \mathbb{C} \rightarrow \mathbb{C}$, with

$$
\Theta(\omega)=\omega+1
$$

and

$$
\varphi(\omega)=\frac{1}{\omega+1}, \quad \varphi(w) \neq 0 .
$$

We check the conditions from the hypothesis of Lemma 1.1. Using (2.17), we have

$$
Q(z, \xi)=z q_{z}^{\prime}(z, \xi) \cdot \varphi(q(z, \xi))=z q_{z}^{\prime}(z, \xi) \cdot \frac{1}{q(z, \xi)+1} .
$$

Differentiating (2.18), and after a short calculus, we obtain

$$
\frac{z Q_{z}^{\prime}(z, \xi)}{Q(z, \xi)}=1+\frac{z q_{z^{2}}^{\prime \prime}(z, \xi)}{q_{z}^{\prime}(z, \xi)}-\frac{z q_{z}^{\prime}(z, \xi)}{q_{z}(z, \xi)+1} .
$$

Using (11) in (2.19), we have

$$
\operatorname{Re} \frac{z Q_{z}^{\prime}(z, \xi)}{Q(z, \xi)}=\operatorname{Re}\left(1+\frac{z q_{z^{2}}^{\prime \prime}(z, \xi)}{q_{z}^{\prime}(z, \xi)}-\frac{z q_{z}^{\prime}(z, \xi)}{q_{z}(z, \xi)+1}\right)>0, \quad z \in U, \xi \in \bar{U}
$$

hence the function $Q(z, \xi)$ is starlike in $U$ for all $\xi \in \bar{U}$. Using (2.16) we have

$$
h(z, \xi)=\theta(q(z, \xi))+Q(z, \xi)=q(z, \xi)+1+Q(z, \xi)
$$

Differentiating (2.21) and using (2.18), (2.20) and (1), after a short calculus, we obtain

$$
\operatorname{Re} \frac{z h_{z}^{\prime}(z, \xi)}{Q(z, \xi)}=\operatorname{Re}\left[q(z, \xi)+1+\frac{z Q_{z}^{\prime}(z, \xi)}{Q(z, \xi)}\right]>0 .
$$

Using (2.16) and (2.17), we get

$$
\begin{array}{ll}
\theta(p(z, \xi))=p(z, \xi)+1, & \varphi(p(z, \xi))=\frac{1}{p(z, \xi)+1}, \\
\theta(q(z, \xi))=q(z, \xi)+1, & \varphi(q(z, \xi))=\frac{1}{q(z, \xi)+1},
\end{array}
$$

and the strong differential subordination (2.12) becomes

$$
\theta(p(z, \xi))+z p_{z}^{\prime}(z, \xi) \cdot \varphi(p(z, \xi)) \prec \prec \theta(q(z, \xi))+z q_{z}^{\prime}(z, \xi) \cdot \varphi(q(z, \xi))
$$

Using Lemma 1.1, we have $p(z, \xi) \prec \prec q(z, \xi)$, i.e., $\frac{\left[H^{m} f(z, \xi)\right]_{z}^{\prime}}{z^{p-1}}-1 \prec \prec q(z, \xi)$ and $q(z, \xi)$ is the best dominant. 
Next we give results related to strong differential superordinations.

Theorem 2.3 Let $h(z, \xi)$ be convex in $U$ for all $\xi \in \bar{U}$, with $h(0, \xi)=a$. Suppose that the differential equation

$$
q(z, \xi)+\frac{z q_{z}^{\prime}(z, \xi)}{q(z, \xi)}=h(z, \xi), \quad z \in U, \xi \in \bar{U}
$$

has a univalent solution $q(z, \xi)$ that satisfies $q(0, \xi)=a$ and $q(z, \xi) \prec \prec h(z, \xi)$.

If $p(z, \xi) \in \mathcal{H}[a, 1] \cap Q_{\xi}$ and $p(z, \xi)+\frac{z p_{z}^{\prime}(z, \xi)}{p(z, \xi)}$ is univalent in $U$ for all $\xi \in \bar{U}, f(z, \xi) \in A \xi$, then

$$
h(z, \xi) \prec \prec \frac{L_{\gamma}^{m} f(z, \xi)}{z}+\frac{z\left(L_{\gamma}^{m} f(z, \xi)\right)_{z}^{\prime}}{L_{\gamma}^{m} f(z, \xi)}-1
$$

implies $q(z, \xi) \prec \prec \frac{L_{\gamma}^{m} f(z, \xi)}{z}, z \in U, \xi \in \bar{U}$. The function $q(z, \xi)$ is the best subordinant.

Proof We let

$$
p(z, \xi)=\frac{L_{\gamma}^{m} f(z, \xi)}{z}, \quad z \in U, \xi \in \bar{U}
$$

From (1.2), we have

$$
p(z, \xi)=\frac{z+\sum_{k=2}^{\infty} \frac{(\gamma+1)^{m}}{(\gamma+k)^{m}} a_{k}(\xi) z^{k}}{z}=1+\sum_{k=2}^{\infty} \frac{(\gamma+1)^{m}}{(\gamma+k)^{m}} a_{k}(\xi) z^{k-1}
$$

and since $p(0, \xi)=1$, we obtain that $p(z, \xi) \in \mathcal{H}[1,1] \cap Q_{\xi}$.

Differentiating (2.26), and after a short calculus, we obtain

$$
p(z, \xi)+\frac{z p_{z}^{\prime}(z, \xi)}{p(z, \xi)}=\frac{z\left(L_{\gamma}^{m} f(z, \xi)\right)_{z}^{\prime}}{L_{\gamma}^{m} f(z, \xi)}+\frac{L_{\gamma}^{m} f(z, \xi)}{z}-1
$$

Using (2.27) in (2.24), the strong differential superordination becomes

$$
q(z, \xi)+\frac{z q_{z}^{\prime}(z, \xi)}{q(z, \xi)} \prec \prec p(z, \xi)+\frac{z p_{z}^{\prime}(z, \xi)}{p(z, \xi)}, \quad z \in U, \xi \in \bar{U}
$$

Using Lemma 1.2, we obtain $q(z, \xi) \prec \prec p(z, \xi)$, i.e., $q(z, \xi) \prec \prec \frac{L_{\gamma}^{m} f(z, \xi)}{z}, z \in U, \xi \in \bar{U}$.

Example 2.1 Let $h(z, \xi)=\frac{1-z \xi}{1+z \xi}, z \in U, \xi \in \bar{U}$, with $\operatorname{Re}\left(1+\frac{z h_{z^{2}}^{\prime \prime}(z, \xi)}{h_{z}^{\prime}(z, \xi)}\right)=\operatorname{Re} \frac{1-z \xi}{1+z \xi}>0, z \in U$, $\xi \in \bar{U}$. From Theorem 2.3 we have that if $m=1, n=1, \gamma=-1+i, f(z, \xi)=z+\frac{\xi}{2} z^{2}$, and $\frac{1+i}{2} z+\frac{1+\xi \frac{1+i}{2} z}{1+\frac{1+i}{2} \frac{\xi}{2} z}$ is univalent in $U$ for $\xi \in \bar{U}$, then

$$
\frac{1-z \xi}{1+z \xi} \prec \prec \frac{1+i}{2} z+\frac{1+\xi \frac{1+i}{2} z}{1+\frac{1+i}{2} \frac{\xi}{2} z}
$$


implies

$$
\frac{1}{1+z \xi} \prec \prec z+\frac{\xi}{2} \frac{1+i}{2} z^{2}, \quad z \in U, \xi \in \bar{U} .
$$

Theorem 2.4 Let $h(z, \xi)$ be convex in $U$ for all $\xi \in \bar{U}$, with $h(0, \xi)=p$. Suppose that the differential equation

$$
q(z, \xi)+\frac{z q_{z}^{\prime}(z, \xi)}{q(z, \xi)}=h(z, \xi), \quad z \in U, \xi \in \bar{U},
$$

has a univalent solution $q(z, \xi)$ that satisfies $q(0, \xi)=p$ and $q(z, \xi) \prec \prec h(z, \xi)$.

If $p(z, \xi) \in \mathcal{H}[p, 1] \cap Q_{\xi}$ and $p(z, \xi)+\frac{z p_{z}^{\prime}(z, \xi)}{p(z, \xi)}$ is univalent in $U$ for all $\xi \in \bar{U}, f(z, \xi) \in A(p) \xi$, then

$$
h(z, \xi) \prec \prec 1+\frac{z^{2}\left(H^{m} f(z, \xi)\right)_{z^{2}}^{\prime \prime}}{\left(H^{m} f(z, \xi)\right)_{z}^{\prime}}, \quad z \in U, \xi \in \bar{U},
$$

implies $q(z, \xi) \prec \prec \frac{z\left(H^{m} f(z, \xi)\right)_{z}^{\prime}}{H^{m} f(z, \xi)}$. The function $q(z, \xi)$ is the best subordinant.

Proof Using (1.5) in (2.29), the strong differential superordination becomes

$$
h(z, \xi) \prec \prec 1+\frac{z\left(H^{m} f(z, \xi)\right)_{z^{2}}^{\prime \prime}}{\left(H^{m} f(z, \xi)\right)_{z}^{\prime}} .
$$

We let

$$
p(z, \xi)=\frac{z\left(H^{m} f(z, \xi)\right)_{z}^{\prime}}{H^{m} f(z, \xi)} .
$$

From (1.4), we have

$$
p(z, \xi)=\frac{z\left(p z^{p-1}+\sum_{k=p+1}^{\infty} \frac{(p+1)^{m}}{(p+k)^{m}} a_{k}(\xi) k z^{k-1}\right)}{z^{p}+\sum_{k=p+1}^{\infty} \frac{(p+1)^{m}}{(p+k)^{m}} a_{k}(\xi) z^{k}}=\frac{p+\sum_{k=p+1}^{\infty} \frac{(p+1)^{m}}{(p+k)^{m}} a_{k}(\xi) z^{k}}{1+\sum_{k=p+1}^{\infty} \frac{(p+1)^{m}}{(p+k)^{m}} a_{k}(\xi) z^{k-p}} .
$$

Since $p(0, \xi)=p$, we obtain that $p(z, \xi) \in \mathcal{H}[p, 1] \cap Q_{\xi}$.

Differentiating (2.31), and after a short calculus, we obtain

$$
p(z, \xi)+\frac{z p_{z}^{\prime}(z, \xi)}{p(z, \xi)}=1+\frac{z\left(H^{m} f(z, \xi)\right)_{z^{2}}^{\prime \prime}}{\left(H^{m} f(z, \xi)\right)_{z}^{\prime}} .
$$

Using (2.32) in (2.30), the strong differential superordination becomes

$$
h(z, \xi) \prec \prec p(z, \xi)+\frac{z p_{z}^{\prime}(z, \xi)}{p(z, \xi)}, \quad z \in U, \xi \in \bar{U} .
$$

Using Lemma 1.2, we obtain $q(z, \xi) \prec \prec p(z, \xi)$, i.e., $q(z, \xi) \prec \prec \frac{z\left(H^{m} f(z, \xi)\right)_{z}^{\prime}}{H^{m} f(z, \xi)}$.

Remark 2.1 Using another integral operator, the author finds interesting results in strong differential subordinations and superordinations in [14]. 


\section{Competing interests}

The author declares that she has no competing interests.

\section{Author's contributions}

The author drafted the manuscript, read and approved the final manuscript.

\section{Acknowledgements}

The author thanks the referee for his/her valuable suggestions to improve the present article.

Received: 11 June 2013 Accepted: 20 September 2013 Published: 08 Nov 2013

\section{References}

1. Miller, SS, Mocanu, PT: Second order differential inequalities in the complex plane. J. Math. Anal. Appl. 65, 298-305 (1978)

2. Miller, SS, Mocanu, PT: Differential subordinations and univalent functions. Mich. Math. J. 28, 157-171 (1981)

3. Miller, SS, Mocanu, PT: Differential Subordinations. Theory and Applications. Pure and Applied Mathematics. Dekker, New York (2000)

4. Miller, SS, Mocanu, PT: Subordinants of differential superordinations. Complex Var. Theory Appl. 48(10), 815-826 (2003)

5. Miller, SS, Mocanu, PT: Briot-Bouquet differential superordinations and sandwich theorems. J. Math. Anal. Appl. 329(1), 327-335 (2007)

6. Antonino, JA, Romaguera, S: Strong differential subordination to Briot-Bouquet differential equations. J. Differ. Equ. 114, 101-105 (1994)

7. Oros, Gl, Oros, G: Strong differential subordination. Turk. J. Math. 33, 249-257 (2009)

8. Oros, GI: On a new strong differential subordination. Acta Univ. Apulensis 32, 6-15 (2012)

9. Oros, Gl: Briot-Bouquet strong differential subordination. J. Comput. Anal. Appl. 14(4), $733-737$ (2012)

10. Alb Lupas, A, Oros, Gl, Oros, G: On special strong differential subordinations using Salagean and Ruscheweyh operators. J. Comput. Anal. Appl. 14(2), 266-270 (2012)

11. Alb Lupas, A: On special strong differential subordinations using multiplier transformation. Appl. Math. Lett. 25 624-630 (2012). doi:10.1016/j.aml.2011.09.074

12. Alb Lupas, A, Oros, Gl, Oros, G: A note on special differential subordinations using multiplier transformation. J. Comput. Anal. Appl. 14(2), 261-265 (2012)

13. Oros, Gl, Oros, G: Second order non-linear strong differential subordinations. Bull. Belg. Math. Soc. Simon Stevin 16(1), 171-178 (2009)

14. Cho, NE: Strong differential subordination properties for analytic functions involving the Komatu integral operator. Bound. Value Probl. 2013, 44 (2013). doi:10.1186/1687-2770-2013-44

15. Oros, Gl: Briot-Bouquet strong differential subordination. J. Comput. Anal. Appl. 14(4), 733-737 (2012)

16. Oros, Gl: Sufficient conditions for univalence obtained by using second order linear strong differential subordinations. Turk. J. Math. 34(1), 13-20 (2010)

17. Sendruţiu, R: Strong differential subordinations obtained by Ruscheweyh operator. J. Comput. Anal. Appl. 14(2), 328-340 (2012)

18. Oros, Gl: Strong differential superordination. Acta Univ. Apulensis 19, 110-116 (2009)

19. Oros, Gl: An application of the subordination chains. Fract. Calc. Appl. Anal. 13(5), 521-530 (2010)

20. Oros, G: Briot-Bouquet strong differential superordinations and sandwich theorems. Math. Rep. 12(62)(3), 277-283 (2010)

21. Oros, Gl: Briot-Bouquet differential subordinations and superordinations using the Dziok-Srivastava linear operator. Math. Rep. 11(2), 155-163 (2009)

22. Oros, Gl, Oros, G: Differential subordinations obtained with some new integral operator (to appear)

10.1186/1687-1847-2013-317

Cite this article as: Oros: Strong differential subordinations and superordinations obtained with some new integral operators. Advances in Difference Equations 2013, 2013:317

\section{Submit your manuscript to a SpringerOpen ${ }^{\ominus}$ journal and benefit from:}

- Convenient online submission

Rigorous peer review

- Immediate publication on acceptance

- Open access: articles freely available online

- High visibility within the field

- Retaining the copyright to your article 